\title{
Evaluation of reduced amino acids diets added with protected protease on productive performance in $25-100 \mathrm{~kg}$ barrows
}

\author{
Jose L. Figueroa ${ }^{a}$, Jose A. Martinez ${ }^{\text {a* }}$, Maria T. Sanchez-Torres ${ }^{\text {a }}$, Jose L. Cordero ${ }^{\text {a }}$, \\ Manuel Martinez ${ }^{\mathrm{b}}$, Victor M. Valdez ${ }^{\mathrm{c}}$, Agustin Ruiz ${ }^{\mathrm{d}}$
}

\begin{abstract}
The objective of this research was to evaluate the effect of adding protected protease to low-amino acids (AA) diets on the growth performance of barrows. Three decreasing levels of AA (protein levels), with or without the addition of protease were fed to 48 hybrid barrows $(27.42 \pm 3.48 \mathrm{~kg}$ initial body weight). The experimental design was a completely randomised with a factorial arrangement of treatments. An analysis of variance was performed with GLM of SAS and the means comparison was performed with Tukey test $(P \leq 0.05)$. The productive performance was not affected by addition of proteases in the diet at the three stages $(P>0.05)$. Only in growing barrows, the interaction of standard protein diet and protease reduced backfat thickness $(P \leq 0.05)$. Protein level in finishing I barrows did not affect $(P>0.05)$ growth performance variables. Low-protein diets increased $(P \leq 0.05)$ average daily gain, final body weight and fat-free lean gain in growing and finishing II barrows. Concentration of urea in plasma decreased with the reduction of $\mathrm{CP}$ and increased with the addition of protease $(P \leq 0.05)$ at the three stages. In conclusion, low protein diets improved or maintained growth performance variables and reduced the plasma urea nitrogen, whereas supplementation with protease did not show any effect on productive performance.

Key words: protein content, swine, growth performance, carcass characteristics.
\end{abstract}

\section{INTRODUCTION}

The use of low-protein diets (LPD) in fattening pigs is a viable option for reducing nitrogen emission into the environment, which reduces the amount of greenhouse gases and soil contamination (Osada et al 2011). In addition, LPD enable to obtain a similar growth performance compared to standard diets when supplemented with crystalline AA (He et al 2016, Jiao et al 2016, Peng et al 2016). However, the reduction of more than 4-6\% of crude protein $(\mathrm{CP})$ in the diet affects growth performance and digestive enzymatic production (He et al 2016). Therefore, it is necessary to find alternative additives that compensate for the reduction of protein ingredients and the amount of $\mathrm{CP}$ in pig diets, increasing AA digestibility.

Addition of protease might improve availability of protein in pig diets through an increment on protein digestibility and amino acid (AA) availability in the gastrointestinal tract of pigs (Wang et al 2011, Guggenbuhl et al 2012). Nevertheless, in some cases, using protease in LPD for weaning, growing and finishing pigs did not improve growth performance or carcass characteristics

Received: 01.08.2018.

Accepted: 22.10.2018.

aPrograma de Ganadería, Colegio de Postgraduados, Montecillo, Texcoco, México.

${ }^{\mathrm{b}}$ Trouw Nutrition Mexico, Parque Industrial Belenes Norte, Zapopan, Jalisco, México.

${ }^{c}$ Animal Nutrition Consultant. Tequexquinahuac, Texcoco, México.

dPosgrado en Producción Animal, Universidad Autónoma Chapingo, Chapingo, México.

*Corresponding author: JA Martinez; Montecillo, Texcoco, Estado de México, CP 56230; alfredo_aispuro@yahoo.com variables (Reyna et al 2006, Zamora et al 2011). The variability in protease effectiveness was attributed to the age of pigs, the use of different types of ingredients, the source of protease (Adeola and Cowieson 2011), and the high degradation and inactivation of these enzymes in the gastrointestinal tract (Pan et al 2016). To surpass this variability of results using protease for pig diets, recent technological advances have developed coated (protected) protease (Xu et al 2017), which works under different conditions in the gastrointestinal tract (Pan et al 2016). Thus, using protected protease in pig diets may improve digestibility of AA which, in turn, is reflected in a better growth performance (Zuo et al 2015).

This improved protein and AA digestibility and availability in the gastrointestinal tract of pigs (Wang et al 2011, Guggenbuhl et al 2012) means that the supplementation of protected protease could compensate for the reduction of AA in diets for pigs when fed LPD. For these reasons, the objective of this research was to evaluate the addition of protected protease to low AA concentration (low-crude protein) diets in $25-100 \mathrm{~kg}$ barrows in terms of growth performance, carcass characteristics, and plasma urea nitrogen concentration.

\section{MATERIAL AND METHODS}

The experimental procedures were performed accordingly to the recommendations of the International Guiding Principles for Biomedical Research Involving Animals ${ }^{1}$, and observing the standards for ethics, biosafety, and animal

\footnotetext{
CIOMS, Council for International Organizations of Medical Sciences. 2012. International Guiding Principles for Biomedical Research Involving Animals. http://www. cioms (Accessed January 23, 2018).
} 
well-being of the Colegio de Postgraduados, Mexico, under the Official Mexican Regulation (Norma Oficial Mexicana, 1999) for the use of animals in experimentation.

The experiment was conducted in the Swine Unit of the Experimental Farm at the Colegio de Postgraduados, located in Montecillo, Estado de Mexico (98 $48^{\circ}$ ' 27' W and $\left.19^{\circ} 48^{\prime} 23^{\prime \prime} \mathrm{N}\right)$. The climate is temperate, semi-arid, with an average annual temperature of $15.9^{\circ} \mathrm{C}$, infrequent frosts, average annual rainfall of $686 \mathrm{~mm}$ and an altitude of $2241 \mathrm{~m}$ (García 1988).

Forty eight hybrid (Landrace $\times$ Yorkshire $\times$ Duroc; $27.42 \pm 3.48 \mathrm{~kg}$ initial body weight and 60 days-old) barrows were used, housed in individual pens, each equipped with a single feeder and nipple drinker. Water and feed were provided ad libitum.

The experiment consisted in the evaluation of six treatments (T). Three decreasing levels of protein (standardized ileal digestible (SID) basis AA levels; control, medium, and low), with or without the addition of protease were evaluated $(0.03 \%$ of protease was added, according to the amount indicated by the JEFO Company; Poultry Grow $250 \AA$, Streptomyces griseus, Type XIV, Puebla, Mexico) in pigs diet. Three stages were evaluated (growing, 25-50 $\mathrm{kg}$ body weight; finishing I, $50-75 \mathrm{~kg}$; finishing II, 75-100 $\mathrm{kg}$; tables 1-3 respectively), changing the feed for each stage accordingly to the average body weight of the pigs, trying to follow the recommendations of the NRC (2012) for each stage. Forty-eight barrows were distributed (each pig was considered a replicate) in a completely randomised design with a factorial arrangement of treatments; there were eight replicates (barrows) per treatment. The AA or CP (low-protein diets; LPD) reduction was achieved by decreasing the lysine content by $0.05 \%$ and $0.10 \%$ relative to control diet, with a proportionate reduction in concentrations of the remaining AA in the diet, trying to maintain the ratio relative to Lys (ensuring that all AA were supplied, at least the Lys proportion).

The nutritional values of the control treatment were established as recommended by the NRC (2012) to cover or exceed the requirements for each stage of growth. The diets for each stage were formulated with the Solver command ${ }^{2}$ using the least-cost feed formulation method.

\section{CHEMICAL ANALYSIS}

Crude protein content of diets for each stage was determined by the Macrokjeldahl method (AOAC 2005), calcium and phosphorus content by atomic absorption spectrophotometry (Karl et al 1979) using a Perkin Elmer 4000 Model (Series Lambda 2, Perkin Elmer Inc., Norwalk, CT, USA). On the last day of the experiment, blood samples ( $5 \mathrm{~mL}$; pre-prandial 08:00 h) were collected by vena cava puncture in live pigs, using Vacutainer tubes

$2 \quad$ Microsoft Excel 2007 without anticoagulant (BD Vacutainer $($ ), and stored at $4{ }^{\circ} \mathrm{C}$. The blood samples were centrifuged (SIGMA 2-16 $\mathrm{k}$, Germany) at $3500 \mathrm{~g}$ for $20 \mathrm{~min}$ to obtain blood serum. Serum samples were stored in Eppendorf tubes at $-20{ }^{\circ} \mathrm{C}$ in a freezer (SANYO MDF-436, USA) until determination of plasma urea (Chaney and Marbach 1962).

\section{VARIABLES}

Growth performance variables were: average daily feed intake (ADFI), average daily gain (ADG), feed:gain ratio (FGR), fat-free lean gain (FFLG) and final body weight (BW). Carcass characteristics determined were: backfat thickness (BT), longissimus muscle area (LMA), lean meat percentage (LMP); and plasma urea nitrogen (PUN) concentration. The BT and LMA were measured using real-time ultrasound (SonoVet 600, MEDISON: Medison, Inc., Cypress, CA, USA) at the 10th rib on the first and last day of the experiment. The BT, LMA, initial and final BW data were used to determine FFLG and LMP, following the procedure indicated by Burson and Berg (2001): Lb. lean $=5.7769+(0.401 \mathrm{x}$ warm carcass wt., lbs $)-(18.838 \mathrm{x}$ 10 th rib fat depth, in. $)+(4.357 \times 10$ th rib loin muscle area, sq. in. $)+(1.006 \times$ sex of pig) $($ barrow $=1$, gilt=2) $)$.

\section{STATISTICAL ANALYSIS}

The experimental design was completely randomised with a factorial arrangement of treatments, where the factors were three CP levels and two protected protease levels (with and without). Shapiro-Wilk and Levene's test were used to check normal distribution and homogeneity of variance for all variables. Data were analyzed with the GLM procedure, and Tukey's test $(P \leq 0.05)$ was used to compare treatment means (Statistical Analysis System 2010. Inc. Cary, NC, USA). The initial body weight was used as a covariate $(P \leq 0.05)$.

\section{RESULTS}

\section{LOW-PROTEIN DIETS}

No differences $(P>0.05)$ were detected between treatments for FGR, LMP, BT and LMA due to the protein level in the diet for growing (table 4) and finishing II barrows (table 6). An improved response was observed in ADG, final BW, ADFI and FFLG in growing barrows fed 16.17 $\%$ and $15.49 \% \mathrm{CP}$ compared to $16.86 \% \mathrm{CP}(P \leq 0.05)$ (table 4). In finishing II barrows, values for ADG and final BW improved $(P \leq 0.05)$ when using $12.08 \% \mathrm{CP}$ in the diet compared to $12.86 \%$ and $13.63 \% \mathrm{CP}$. The FFLG was greater $(P \leq 0.05)$ when using $12.86 \%$ and $12.08 \%$ $\mathrm{CP}$ compared to control diet $(13.63 \% \mathrm{CP})$ (table 6$)$. There were no effects $(P>0.05)$ of protein level on any growth performance variables in finishing I $(50-75 \mathrm{~kg})$ barrows (table 5). 
PROTEIN CONTENT, SWINE, GROWTH PERFORMANCE, CARCASS CHARACTERISTICS

Table 1. Composition of diets for growing barrows (25-50 kg BW) fed low-protein diets supplemented with protease.

\begin{tabular}{|c|c|c|c|c|c|c|}
\hline Ingredient, \% & $\mathrm{T} 1^{\mathrm{II}}$ & $\mathrm{T} 2$ & $\mathrm{~T} 3$ & $\mathrm{~T} 4$ & T5 & T6 \\
\hline Sorghum grain (Sorghum bicolor) & 77.55 & 77.51 & 79.35 & 79.31 & 81.11 & 81.06 \\
\hline Soybean (Glycine max) meal & 18.66 & 18.66 & 16.90 & 16.91 & 15.19 & 15.20 \\
\hline Soybean oil & 0.97 & 0.99 & 0.94 & 0.96 & 0.91 & 0.92 \\
\hline Biolys $^{\dagger}$ & 0.73 & 0.73 & 0.72 & 0.72 & 0.71 & 0.71 \\
\hline DL-Methionine & 0.16 & 0.16 & 0.14 & 0.14 & 0.13 & 0.13 \\
\hline L-Threonine & 0.10 & 0.10 & 0.09 & 0.09 & 0.09 & 0.09 \\
\hline Vitamins $^{\S}$ & 0.20 & 0.20 & 0.20 & 0.20 & 0.20 & 0.20 \\
\hline Minerals $^{\mathrm{P}}$ & 0.15 & 0.15 & 0.15 & 0.15 & 0.15 & 0.15 \\
\hline Salt & 0.30 & 0.30 & 0.30 & 0.30 & 0.30 & 0.30 \\
\hline Calcium carbonate & 0.91 & 0.91 & 0.89 & 0.89 & 0.87 & 0.87 \\
\hline Calcium orthophosphate & 0.26 & 0.26 & 0.29 & 0.30 & 0.33 & 0.33 \\
\hline Phytase & 0.01 & 0.01 & 0.01 & 0.01 & 0.01 & 0.01 \\
\hline Protease & 0.00 & 0.03 & 0.00 & 0.03 & 0.00 & 0.03 \\
\hline Total & 100.00 & 100.00 & 100.00 & 100.00 & 100.00 & 100.00 \\
\hline \multicolumn{7}{|c|}{ Nutrient composition calculated (SID AA) } \\
\hline Metabolizable Energy $\left(\right.$ Mcal kg$^{-1}$ ) & 3.30 & 3.30 & 3.30 & 3.30 & 3.30 & 3.30 \\
\hline Crude Protein (\%) & 16.86 & 16.86 & 16.17 & 16.17 & 15.49 & 15.49 \\
\hline Lysine (\%) & 1.00 & 1.00 & 0.95 & 0.95 & 0.90 & 0.90 \\
\hline Threonine (\%) & 0.60 & 0.60 & 0.57 & 0.57 & 0.54 & 0.54 \\
\hline Tryptophan (\%) & 0.17 & 0.17 & 0.16 & 0.16 & 0.15 & 0.15 \\
\hline Phenialanine (\%) & 0.73 & 0.73 & 0.70 & 0.70 & 0.67 & 0.67 \\
\hline Arginine (\%) & 0.87 & 0.87 & 0.81 & 0.81 & 0.76 & 0.76 \\
\hline Histidine (\%) & 0.36 & 0.36 & 0.34 & 0.34 & 0.32 & 0.32 \\
\hline Isoleucine (\%) & 0.61 & 0.61 & 0.58 & 0.58 & 0.55 & 0.55 \\
\hline Leucine $(\%)$ & 1.45 & 1.45 & 1.41 & 1.41 & 1.38 & 1.38 \\
\hline Valine $(\%)$ & 0.76 & 0.76 & 0.73 & 0.73 & 0.69 & 0.69 \\
\hline Methionine + Cysteine (\%) & 0.57 & 0.57 & 0.54 & 0.54 & 0.51 & 0.51 \\
\hline Calcium (\%) & 0.67 & 0.67 & 0.67 & 0.67 & 0.67 & 0.67 \\
\hline Phosphorus (\%) & 0.40 & 0.40 & 0.40 & 0.40 & 0.40 & 0.40 \\
\hline \multicolumn{7}{|c|}{ Determined nutrient composition } \\
\hline $\mathrm{CP}(\%)$ & 16.76 & 16.74 & 16.09 & 16.07 & 15.39 & 15.42 \\
\hline Calcium (\%) & 0.72 & 0.73 & 0.71 & 0.75 & 0.74 & 0.73 \\
\hline Phosphorus (\%) & 0.38 & 0.37 & 0.39 & 0.38 & 0.37 & 0.36 \\
\hline
\end{tabular}

${ }^{\dagger}$ Biolys, 50.7\%; lysine. ${ }^{\mathrm{I} T}$, Treatment. §Supplied by kg: 5.0×106 IU vitamin A, 1.0×106 IU vitamin D3, $2.0 \times 104$ IU vitamin E; 2 g vitamin K3, 1 g tiamine, $5 \mathrm{~g}$ rivoflavin, $2 \mathrm{~g}$ pyridoxine, $25 \mathrm{~g}$ niacin, $15 \mathrm{~g}$ D-calcium panthotenate, $3 \mathrm{~g}$ folic acid, $225 \mathrm{~g}$ choline chloride, $0.3 \mathrm{~g}$ antioxidant, $15 \mathrm{mg}$ vitamin $\mathrm{B} 12$ and $180 \mathrm{mg}$ vitamin H-biotin. REKA® Lapisa Animal Nutrition. ${ }^{\mathrm{P} S}$ Supplied by kg: $0.2 \mathrm{~g} \mathrm{Se}, 0.1 \mathrm{~g} \mathrm{Co}, 0.3 \mathrm{~g} \mathrm{I}, 10 \mathrm{~g} \mathrm{Cu}, 100 \mathrm{~g}$ Zn, $100 \mathrm{~g}$ Fe and $100 \mathrm{~g}$ Mn. REKA® Lapisa Animal Nutrition.

\section{PROTEASE}

The addition of protease to diets during three growth phases (growing, finishing I and finishing II) did not change $(P>0.05)$ the growth performance and carcass characteristics of barrows (tables 4, 5 and 6, respectively).

In the growing stage, the control diet $(16.86 \% \mathrm{CP})$ with added protease reduced BT $(P \leq 0.05)$; the protease interaction with the $\mathrm{CP}$ level had no effect $(\mathrm{P}>0.05)$ on other variables (table 4$)$. There were no effects $(P>0.05)$ of the interaction between protein level and protease addition on growth performance and carcass characteristics in finishing I and finishing II barrows (tables 5 and 6).

\section{PLASMA UREA NITROGEN}

The addition of dietary protease in the three phases of growth increased PUN concentration $(P \leq 0.05)$ of barrows; while protein reduction reduced PUN concentration in all three phases $(P \leq 0.05)$. In growing barrows, the lowest 
Table 2. Composition of diets for finishing I barrows (50-75 kg BW) fed low-protein diets supplemented with protease.

\begin{tabular}{|c|c|c|c|c|c|c|}
\hline Ingredient, \% & $\mathrm{T} 1^{\mathbb{I}}$ & $\mathrm{T} 2$ & $\mathrm{~T} 3$ & $\mathrm{~T} 4$ & T5 & T6 \\
\hline Sorghum grain & 81.80 & 81.80 & 83.88 & 83.83 & 85.91 & 85.86 \\
\hline Soybean meal & 14.58 & 14.58 & 12.57 & 12.58 & 10.56 & 10.57 \\
\hline Soybean oil & 0.91 & 0.91 & 0.86 & 0.87 & 0.82 & 0.84 \\
\hline Biolys $^{\dagger}$ & 0.64 & 0.64 & 0.64 & 0.64 & 0.65 & 0.65 \\
\hline DL-Methionine & 0.10 & 0.10 & 0.09 & 0.09 & 0.08 & 0.08 \\
\hline L-Threonine & 0.07 & 0.07 & 0.07 & 0.07 & 0.07 & 0.07 \\
\hline Vitamins ${ }^{\S}$ & 0.20 & 0.20 & 0.20 & 0.20 & 0.20 & 0.20 \\
\hline Minerals $^{\mathrm{P}}$ & 0.15 & 0.15 & 0.15 & 0.15 & 0.15 & 0.15 \\
\hline Salt & 0.30 & 0.30 & 0.30 & 0.30 & 0.30 & 0.30 \\
\hline Calcium carbonate & 0.87 & 0.87 & 0.84 & 0.84 & 0.82 & 0.82 \\
\hline Calcium orthophosphate & 0.34 & 0.34 & 0.39 & 0.39 & 0.43 & 0.43 \\
\hline Phytases & 0.01 & 0.01 & 0.01 & 0.01 & 0.01 & 0.01 \\
\hline Protease & 0.00 & 0.03 & 0.00 & 0.03 & 0.00 & 0.03 \\
\hline Total & 100.00 & 100.00 & 100.00 & 100.00 & 100.00 & 100.00 \\
\hline \multicolumn{7}{|c|}{ Nutrient composition calculated (SID AA) } \\
\hline Metabolizable Energy $\left(\mathrm{Mcal} \mathrm{kg}^{-1}\right)$ & 3.30 & 3.30 & 3.30 & 3.30 & 3.30 & 3.30 \\
\hline Crude Protein $(\%)$ & 15.18 & 15.18 & 14.40 & 14.40 & 13.63 & 13.63 \\
\hline Lysine $(\%)$ & 0.85 & 0.85 & 0.80 & 0.80 & 0.75 & 0.75 \\
\hline Threonine (\%) & 0.52 & 0.52 & 0.49 & 0.49 & 0.46 & 0.46 \\
\hline Tryptophan (\%) & 0.15 & 0.15 & 0.14 & 0.14 & 0.13 & 0.13 \\
\hline Phenialanine (\%) & 0.66 & 0.66 & 0.63 & 0.63 & 0.59 & 0.59 \\
\hline Arginine $(\%)$ & 0.75 & 0.75 & 0.69 & 0.69 & 0.63 & 0.63 \\
\hline Histidine (\%) & 0.32 & 0.32 & 0.30 & 0.30 & 0.28 & 0.28 \\
\hline Isoleucine $(\%)$ & 0.54 & 0.54 & 0.51 & 0.51 & 0.48 & 0.48 \\
\hline Leucine $(\%)$ & 1.36 & 1.36 & 1.32 & 1.32 & 1.28 & 1.28 \\
\hline Valine $(\%)$ & 0.68 & 0.68 & 0.64 & 0.64 & 0.60 & 0.60 \\
\hline Methionine+Cysteine (\%) & 0.48 & 0.48 & 0.45 & 0.45 & 0.42 & 0.42 \\
\hline Calcium $(\%)$ & 0.62 & 0.62 & 0.62 & 0.62 & 0.62 & 0.62 \\
\hline Phosphorus (\%) & 0.33 & 0.33 & 0.33 & 0.33 & 0.33 & 0.33 \\
\hline \multicolumn{7}{|c|}{ Nutrient composition (evaluated in laboratory) } \\
\hline Crude Protein $(\%)$ & 15.12 & 15.16 & 14.24 & 14.32 & 13.57 & 13.52 \\
\hline Calcium (\%) & 0.71 & 0.72 & 0.75 & 0.73 & 0.70 & 0.73 \\
\hline Phosphorus (\%) & 0.36 & 0.37 & 0.38 & 0.37 & 0.34 & 0.39 \\
\hline
\end{tabular}

${ }^{\dagger}$ Biolys, 50.7\%; lysine. ${ }^{\mathrm{I}} \mathrm{T}$, Treatment. ${ }^{\S}$ Supplied by kg: 5.0×106 IU vitamin A, 1.0×106 IU vitamin D3, $2.0 \times 104$ IU vitamin E; 2 g vitamin K3, 1 g tiamine, $5 \mathrm{~g}$ rivoflavin, $2 \mathrm{~g}$ pyridoxine, $25 \mathrm{~g}$ niacin, $15 \mathrm{~g}$ D-calcium panthotenate, $3 \mathrm{~g}$ folic acid, $225 \mathrm{~g}$ choline chloride, $0.3 \mathrm{~g}$ antioxidant, $15 \mathrm{mg}$ vitamin B12 and $180 \mathrm{mg}$ vitamin H-biotin. REKA® Lapisa Animal Nutrition. ${ }^{\mathrm{P} S}$ Supplied by kg: $0.2 \mathrm{~g} \mathrm{Se}, 0.1 \mathrm{~g} \mathrm{Co}, 0.3 \mathrm{~g} \mathrm{I}, 10 \mathrm{~g}$ Cu, $100 \mathrm{~g}$ Zn, $100 \mathrm{~g}$ Fe and $100 \mathrm{~g} \mathrm{Mn.} \mathrm{REKA®} \mathrm{Lapisa} \mathrm{Animal} \mathrm{Nutrition.}$

concentration of this metabolite occurred with the lowest CP level $(15.49 \%)$ and the addition of protease $(P \leq 0.05)$. In finishing I barrows, the lowest PUN concentration was also detected with the lowest CP concentration (13.63 $\%)$ regardless the addition or not of protease $(P \leq 0.05)$. Finishing II barrows showed lower PUN $(P \leq 0.05)$ with the lowest PC concentration $(12.08 \%)$ as in the previous growth stages.

\section{DISCUSSION}

The NRC (2012) does not establish a CP value for diets formulation, so, its concentration in the diet is the result of the AA level. The concentrations of lysine, methionine, threonine, tryptophan and other AA were reduced in the diets used in this study, trying to maintain the proportion of AA with respect to lysine (tables 1-3), which reduced the CP level of the diet. However, under the concept of minimum cost formulation of diets for pigs, in economic terms it is more feasible to exceed the recommendation 
Table 3. Composition of diets for finishing II barrows (75-100 kg BW) fed low-protein diets supplemented with protease.

\begin{tabular}{|c|c|c|c|c|c|c|}
\hline Ingredient, $\%$ & $\mathrm{~T} 1^{\mathrm{qI}}$ & $\mathrm{T} 2$ & $\mathrm{~T} 3$ & $\mathrm{~T} 4$ & T5 & T6 \\
\hline Sorghum grain & 87.03 & 86.98 & 89.10 & 89.05 & 91.16 & 91.11 \\
\hline Soybean meal & 10.41 & 10.42 & 8.40 & 8.41 & 6.39 & 6.40 \\
\hline Soybean oil & 0.45 & 0.47 & 0.40 & 0.42 & 0.35 & 0.37 \\
\hline Biolys $^{\dagger}$ & 0.61 & 0.61 & 0.61 & 0.61 & 0.61 & 0.61 \\
\hline DL-Methionine & 0.08 & 0.08 & 0.07 & 0.07 & 0.06 & 0.06 \\
\hline L-Threonine & 0.07 & 0.07 & 0.06 & 0.06 & 0.06 & 0.06 \\
\hline Vitamins $^{\S}$ & 0.20 & 0.20 & 0.20 & 0.20 & 0.20 & 0.20 \\
\hline Minerals $^{\mathrm{P}}$ & 0.15 & 0.15 & 0.15 & 0.15 & 0.15 & 0.15 \\
\hline Salt & 0.30 & 0.30 & 0.30 & 0.30 & 0.30 & 0.30 \\
\hline Calcium carbonate & 0.69 & 0.69 & 0.70 & 0.70 & 0.70 & 0.70 \\
\hline Phytase & 0.01 & 0.01 & 0.01 & 0.01 & 0.01 & 0.01 \\
\hline Protease & 0.00 & 0.03 & 0.00 & 0.03 & 0.00 & 0.03 \\
\hline Total & 100.00 & 100.00 & 100.00 & 100.00 & 100.00 & 100.00 \\
\hline \multicolumn{7}{|c|}{ Nutrient composition calculated (SID AA) } \\
\hline Metabolizable Energy (Mcal kg-1) & 3.30 & 3.30 & 3.30 & 3.3 & 3.30 & 3.3 \\
\hline Crude Protein $(\%)$ & 13.63 & 13.63 & 12.86 & 12.85 & 12.08 & 12.08 \\
\hline Lysine $(\%)$ & 0.73 & 0.73 & 0.68 & 0.68 & 0.63 & 0.63 \\
\hline Threonine (\%) & 0.46 & 0.46 & 0.43 & 0.43 & 0.40 & 0.40 \\
\hline Tryptophan (\%) & 0.13 & 0.13 & 0.12 & 0.12 & 0.11 & 0.11 \\
\hline Phenialanine (\%) & 0.59 & 0.59 & 0.56 & 0.56 & 0.53 & 0.53 \\
\hline Arginine (\%) & 0.63 & 0.63 & 0.57 & 0.57 & 0.51 & 0.51 \\
\hline Histidine (\%) & 0.28 & 0.28 & 0.26 & 0.26 & 0.24 & 0.24 \\
\hline Isoleucine $(\%)$ & 0.48 & 0.48 & 0.45 & 0.45 & 0.41 & 0.41 \\
\hline Leucine $(\%)$ & 1.29 & 1.29 & 1.24 & 1.24 & 1.20 & 1.20 \\
\hline Valine $(\%)$ & 0.60 & 0.60 & 0.56 & 0.56 & 0.52 & 0.52 \\
\hline Methionine + Cysteine $(\%)$ & 0.42 & 0.42 & 0.39 & 0.39 & 0.36 & 0.36 \\
\hline Calcium $(\%)$ & 0.52 & 0.52 & 0.52 & 0.52 & 0.52 & 0.52 \\
\hline Phosphorus (\%) & 0.32 & 0.32 & 0.32 & 0.32 & 0.31 & 0.31 \\
\hline \multicolumn{7}{|c|}{ Nutrient composition (evaluated in laboratory) } \\
\hline Crude Protein $(\%)$ & 13.43 & 13.41 & 12.92 & 12.85 & 12.01 & 12.10 \\
\hline Calcium $(\%)$ & 0.52 & 0.54 & 0.51 & 0.53 & 0.52 & 0.53 \\
\hline Phosphorus (\%) & 0.37 & 0.36 & 0.39 & 0.33 & 0.34 & 0.36 \\
\hline
\end{tabular}

${ }^{\dagger}$ Biolys, 50.7\%; lysine. ${ }^{\text {ITT, Treatment. }}{ }^{\S}$ Supplied by kg: 5.0×106 IU vitamin A, 1.0×106 IU vitamin D3, 2.0×104 IU vitamin E; $2 \mathrm{~g}$ vitamin K3, $1 \mathrm{~g}$ tiamine, $5 \mathrm{~g}$ rivoflavin, $2 \mathrm{~g}$ pyridoxine, $25 \mathrm{~g}$ niacin, $15 \mathrm{~g}$ D-calcium panthotenate, $3 \mathrm{~g}$ folic acid, $225 \mathrm{~g}$ choline chloride, $0.3 \mathrm{~g}$ antioxidant, $15 \mathrm{mg}$ vitamin $\mathrm{B} 12$ and $180 \mathrm{mg}$ vitamin H-biotin. REKA® Lapisa Animal Nutrition. ${ }^{\mathrm{P} S u p p l i e d ~ b y ~ k g: ~} 0.2 \mathrm{~g} \mathrm{Se}, 0.1 \mathrm{~g} \mathrm{Co}, 0.3 \mathrm{~g} \mathrm{I}, 10 \mathrm{~g} \mathrm{Cu}, 100 \mathrm{~g} \mathrm{Zn}, 100 \mathrm{~g}$ Fe and 100 g Mn. REKA® Lapisa Animal Nutrition.

of some AA (NRC 2012) than trying to accomplish the lower (goal) values (Dubeau et al 2011). In our study, the reduction of phenylalanine, arginine, isoleucine, leucine and valine was limited because the basal ingredients (sorghum grain-soybean meal) have a high concentration of these AA.

\section{LOW-PROTEIN DIETS}

The results indicate that it is feasible to improve or maintain growth performance variables when the amount of AA in barrow diets is reduced, as long as the lysine:AA ratio is maintained. Results of other studies showed that finishing pigs fed LPD, with an unchanged AA ratio per $\mathrm{CP}$ unit, had similar and even improved growth variables (Gallo et al 2014, Tous et al 2014).

In contrast, Qin et al (2015), Jiao et al (2016) and Peng et al (2016) reported that these growth variables were unaffected when $\mathrm{CP}$ content was reduced, but the requirement for the more limiting AA (Lys, Met, Trp, Thr) must be reached in the diet. The use of lower levels of AA in our experiment with a performance similar to that of the control protein diet, leads us to determine that 
Table 4. Productive performance, carcass characteristics and plasma urea nitrogen concentration of growing barrows (25-50 kg) fed three levels of crude protein and two levels of protease.

\begin{tabular}{|c|c|c|c|c|c|c|c|c|c|c|c|}
\hline$\% \mathrm{CP}$ & Protease & $\begin{array}{c}\text { ADFI } \\
\left(\mathrm{kg} \mathrm{d}^{-1}\right)\end{array}$ & $\begin{array}{c}\text { ADG } \\
\left(\mathrm{kg} \mathrm{d}^{-1}\right)\end{array}$ & FGR & $\begin{array}{l}\text { BWi } \\
(\mathrm{kg})\end{array}$ & $\begin{array}{l}\text { BWf } \\
(\mathrm{kg})\end{array}$ & $\begin{array}{c}\text { FFLG } \\
\left(\mathrm{kg} \mathrm{d}^{-1}\right)\end{array}$ & $\begin{array}{c}\text { LMP } \\
(\%)\end{array}$ & $\begin{array}{l}\text { BT } \\
(\mathrm{mm})\end{array}$ & $\begin{array}{l}\text { LMA } \\
\left(\mathrm{cm}^{2}\right)\end{array}$ & $\begin{array}{c}\text { PUN } \\
\left(\mathrm{mg} \mathrm{dL}^{-1}\right)\end{array}$ \\
\hline 16.86 & - & 1.67 & 0.70 & 2.44 & 26.62 & 49.03 & 0.251 & 30.07 & $6.22^{\mathrm{a}}$ & 19.36 & $21.26^{\mathrm{b}}$ \\
\hline 16.86 & + & 1.53 & 0.64 & 2.42 & 27.76 & 47.20 & 0.236 & 30.65 & $5.20^{\mathrm{b}}$ & 18.86 & $27.93^{\mathrm{a}}$ \\
\hline 16.17 & - & 1.74 & 0.77 & 2.26 & 27.58 & 51.51 & 0.287 & 30.07 & $5.84^{\mathrm{ab}}$ & 19.86 & $18.73^{\mathrm{bc}}$ \\
\hline 16.17 & + & 1.79 & 0.75 & 2.43 & 27.27 & 50.56 & 0.274 & 30.17 & $6.16^{\mathrm{a}}$ & 19.77 & $21.10^{\mathrm{b}}$ \\
\hline 15.49 & - & 1.87 & 0.72 & 2.63 & 27.75 & 49.82 & 0.267 & 29.97 & $6.05^{\mathrm{ab}}$ & 19.37 & $12.43^{\mathrm{d}}$ \\
\hline 15.49 & + & 1.85 & 0.76 & 2.44 & 27.47 & 50.93 & 0.279 & 29.90 & $5.87^{\mathrm{ab}}$ & 19.35 & $16.54^{\mathrm{c}}$ \\
\hline SEM & & 0.07 & 0.03 & 0.11 & 1.30 & 0.91 & 0.01 & 0.39 & 0.26 & 0.77 & 0.80 \\
\hline \multicolumn{12}{|l|}{$P$ value } \\
\hline$\% \mathrm{CP} \times$ Protease & & 0.39 & 0.26 & 0.26 & - & 0.26 & 0.51 & 0.68 & 0.04 & 0.94 & 0.03 \\
\hline$\% \mathrm{CP}$ & & 0.002 & 0.005 & 0.22 & - & 0.005 & 0.01 & 0.54 & 0.48 & 0.65 & 0.001 \\
\hline Protease & & 0.51 & 0.45 & 0.87 & - & 0.452 & 0.61 & 0.53 & 0.18 & 0.75 & 0.001 \\
\hline
\end{tabular}

$\mathrm{CP}=$ Crude Protein $; \mathrm{ADFI}=$ average daily feed intake $\mathrm{ADG}=$ average daily gain $; \mathrm{FGR}=$ feed : gain ratio; $\mathrm{BWi}=$ initial body weight $\mathrm{BWf}=$ final body weight; FFLG= Fat free lean gain; LMP= lean meat percentage; BT= backfat thickness; LMA= Longissimus muscle area; $\mathrm{PUN}=$ plasma urea nitrogen concentration, SEM= standard error of the mean. ${ }^{\mathrm{a}, \mathrm{b}, \mathrm{c}, \mathrm{d}}$ Means with different superscript differ $(P \leq 0.05)$.

Table 5. Productive performance, carcass characteristics and plasma urea nitrogen concentration of finishing I barrows (50-75 kg) fed three levels of protein and two levels of protease.

\begin{tabular}{lccccccccccc}
\hline \% CP & Protease & $\begin{array}{c}\text { ADFI } \\
\left(\mathrm{kg} \mathrm{d}^{-1}\right)\end{array}$ & $\begin{array}{c}\text { ADG } \\
\left(\mathrm{kg} \mathrm{d}^{-1}\right)\end{array}$ & FGR & $\begin{array}{c}\text { BWi } \\
(\mathrm{kg})\end{array}$ & $\begin{array}{c}\mathrm{BWf} \\
(\mathrm{kg})\end{array}$ & $\begin{array}{c}\text { FFLG } \\
\left(\mathrm{kg} \mathrm{d}^{-1}\right)\end{array}$ & $\begin{array}{c}\text { LMP } \\
(\%)\end{array}$ & $\begin{array}{c}\text { BT } \\
\left(\mathrm{mm}^{2}\right)\end{array}$ & $\begin{array}{c}\text { LMA } \\
\left(\mathrm{cm}^{2}\right)\end{array}$ & $\begin{array}{c}\text { PUN } \\
\left(\mathrm{mg} \mathrm{dL}^{-1}\right)\end{array}$ \\
\hline 15.18 & - & 2.63 & 0.94 & 2.81 & 47.77 & 76.44 & 0.311 & 28.12 & 10.34 & 25.74 & $23.50^{\mathrm{b}}$ \\
15.18 & + & 2.57 & 0.93 & 2.78 & 49.08 & 76.02 & 0.307 & 28.33 & 9.56 & 25.52 & $30.66^{\mathrm{a}}$ \\
14.40 & - & 2.46 & 0.93 & 2.67 & 51.77 & 76.03 & 0.307 & 28.21 & 9.64 & 25.33 & $22.57^{\mathrm{b}}$ \\
14.40 & + & 2.42 & 0.87 & 2.83 & 50.31 & 74.35 & 0.290 & 28.34 & 10.09 & 25.59 & $28.42^{\mathrm{a}}$ \\
13.63 & - & 2.67 & 0.90 & 3.00 & 50.33 & 75.17 & 0.298 & 28.34 & 10.08 & 25.45 & $17.45^{\mathrm{c}}$ \\
13.63 & + & 2.51 & 0.89 & 2.84 & 50.98 & 75.07 & 0.297 & 28.19 & 9.74 & 25.04 & $17.90^{\mathrm{c}}$ \\
SEM & & 0.09 & 0.04 & 0.11 & 2.25 & 1.20 & 0.011 & 0.23 & 0.25 & 0.75 & 0.93 \\
$P$ value & & & & & & & & & & & \\
$\%$ CP $\times$ Protease & & 0.52 & 0.94 & 0.41 & - & 0.95 & 0.94 & 0.92 & 0.08 & 0.93 & 0.001 \\
$\%$ CP & & 0.19 & 0.90 & 0.60 & - & 0.91 & 0.90 & 0.98 & 0.95 & 0.94 & 0.001 \\
Protease & & 0.43 & 0.67 & 0.76 & - & 0.66 & 0.67 & 0.73 & 0.31 & 0.96 & 0.001 \\
\hline
\end{tabular}

$\mathrm{CP}=$ Crude Protein; $\mathrm{ADFI}=$ average daily feed intake; $\mathrm{ADG}=$ average daily gain; $\mathrm{FGR}=$ feed:gain ratio; $\mathrm{BWi}=$ initial body weight; $\mathrm{BWf}=$ final body weight; FFLG= Fat free lean gain; LMP= lean meat percentage; BT= backfat thickness; LMA= Longissimus muscle area; PUN= plasma urea nitrogen concentration, SEM= standard error of the mean. ${ }^{\mathrm{a}, \mathrm{b}, \mathrm{c}, \mathrm{d}}$ Means with different superscript differ $(P \leq 0.05)$.

the nutritional requirement values coincide with the recommendations for pigs with low genetic potential for lean growth, when comparing $\mathrm{CP}$ and lysine concentrations evaluated in our study with the NRC (2012) and Brazilian tables (Rostagno et al 2017).

In the present research, the decrease in $\mathrm{CP}$ as a result of AA reduction showed beneficial or null effects on the growth performance. Gloaguen et al (2014) and González et al (2016) reported that a drastic reduction in dietary CP should not be made, because lowering CP by more than three percentage units affected ADG, ADFI, FGR, final BW and FFLG. The negative response to LPD may be attributed to AA deficiency, because $\mathrm{CP}$ reduction limits the concentration of AA and the amount of nitrogen necessary for the synthesis of non-essential AA (Gloaguen et al 2014).

Consistent with results of our study, Zamora et al (2011) and Qin et al (2015) confirmed that decreasing CP by less than two percentage units in diets for fattening pigs did not affect carcass characteristics. This may be a result of better balance of AA for protein synthesis and, therefore, AA are not required as an energy source, reflected by similar LMP values with different CP concentrations (Orlando et al 2007). However, González et al (2016) and Figueroa et al (2012) observed that in nursery and finishing pigs, decreasing dietary $\mathrm{CP}$ by more than three 
Table 6. Productive performance, carcass characteristics and plasma urea nitrogen concentration of finishing II barrows (75-100 kg) fed with three levels of protein and two levels of protease.

\begin{tabular}{|c|c|c|c|c|c|c|c|c|c|c|c|}
\hline$\% \mathrm{CP}$ & Protease & $\begin{array}{c}\text { ADFI } \\
\left(\mathrm{kg} \mathrm{d}^{-1}\right)\end{array}$ & $\begin{array}{c}\text { ADG } \\
\left(\mathrm{kg} \mathrm{d}^{-1}\right)\end{array}$ & FGR & $\begin{array}{l}\text { BWi } \\
(\mathrm{kg})\end{array}$ & $\begin{array}{l}\text { BWf } \\
(\mathrm{kg})\end{array}$ & $\begin{array}{c}\text { FFLG } \\
\left(\mathrm{kg} \mathrm{d}^{-1}\right)\end{array}$ & $\begin{array}{l}\text { LMP } \\
(\%)\end{array}$ & $\begin{array}{c}\text { BT } \\
(\mathrm{mm})\end{array}$ & $\begin{array}{l}\text { LMA } \\
\left(\mathrm{cm}^{2}\right)\end{array}$ & $\begin{array}{c}\text { PUN } \\
\left(\mathrm{mg} \mathrm{dL}^{-1}\right)\end{array}$ \\
\hline 13.63 & - & 3.19 & 0.85 & 3.87 & 76.44 & 102.77 & 0.321 & 28.10 & 16.70 & 36.83 & $17.64^{\mathrm{a}}$ \\
\hline 13.63 & + & 3.59 & 0.96 & 3.74 & 76.02 & 106.32 & 0.357 & 28.12 & 14.52 & 36.02 & $19.47^{\mathrm{a}}$ \\
\hline 12.86 & - & 3.16 & 0.92 & 3.44 & 76.03 & 104.97 & 0.358 & 28.41 & 13.96 & 36.58 & $16.69^{\mathrm{a}}$ \\
\hline 12.86 & + & 3.39 & 0.88 & 3.91 & 74.35 & 103.72 & 0.336 & 28.32 & 15.85 & 37.30 & $18.97^{\mathrm{a}}$ \\
\hline 12.08 & - & 3.55 & 1.01 & 3.53 & 75.17 & 108.00 & 0.380 & 28.19 & 15.18 & 36.92 & $13.36^{\mathrm{b}}$ \\
\hline 12.08 & + & 3.51 & 1.02 & 3.44 & 75.07 & 108.20 & 0.365 & 27.69 & 16.33 & 36.26 & $17.51^{\mathrm{a}}$ \\
\hline SEM & & 0.15 & 0.04 & 0.17 & 1.20 & 1.38 & 0.015 & 0.23 & 0.07 & 0.80 & 0.80 \\
\hline \multicolumn{12}{|l|}{$P$ value } \\
\hline$\% \mathrm{CP} \times$ Protease & & 0.22 & 0.20 & 0.15 & - & 0.20 & 0.14 & 0.51 & 0.10 & 0.57 & 0.05 \\
\hline$\% \mathrm{CP}$ & & 0.22 & 0.01 & 0.17 & - & 0.01 & 0.05 & 0.19 & 0.46 & 0.80 & 0.001 \\
\hline Protease & & 0.10 & 0.46 & 0.54 & - & 0.45 & 0.97 & 0.31 & 0.62 & 0.69 & 0.001 \\
\hline
\end{tabular}

$\mathrm{CP}=$ Crude Protein $; \mathrm{ADFI}=$ average daily feed intake; $\mathrm{ADG}=$ average daily gain $; \mathrm{FGR}=$ feed:gain ratio $\mathrm{BWi}=$ initial body weight; $\mathrm{BWf}=$ final body weight; FFLG= Fat free lean gain; LMP= lean meat percentage; BT= backfat thickness; LMA= Longissimus muscle area; PUN= plasma urea nitrogen concentration, SEM= standard error of the mean. ${ }^{\text {a,b,c,d }}$ Means with different superscript differ $(P \leq 0.05)$.

percentage units had a negative effect on BT, LMP and LMA. Reduction in FFLG and increase in BT in pigs fed LPD were attributed to the greater availability of energy for adipose tissue accretion, due to an imbalance between a greater amount of available energy and a deficiency of AA (Gómez et al 2002).

\section{PROTEASE}

Various reports (Zuo et al 2015, Pan et al 2016, Yu et al 2016) have demonstrated the efficacy of adding protected protease to pig diets, increasing CP digestibility and AA availability, improvements in the plasma concentration of total protein, increased pepsin enzyme activity in the stomach, as well as increased pancreatic amylase and trypsin. The combination of an increase in $\mathrm{CP}$ digestibility and lower consumption could reflect improvements in the growth performance of pigs fed protease (Mc Alpine et al 2012, O'Shea et al 2014). These changes resulted in a better productive performance in both weaning and nursery pigs, which does not have adequate protease production (Zuo et al 2015, Greiner et al 2016).

Previous research showed that supplementation of LPD with protease did not improve growth performance in fattening pigs (Morales et al 2002, Reyna et al 2006, Zamora et al 2011). Besides, Mc Alpine et al (2012) and O'Shea et al (2014) observed that the use of protected protease in pig diets could reduce ADG in response to a reduction of ADFI.

This phenomenon could be explained because the protease hydrolyses the protein in the small intestine, which releases components that can be absorbed, and increases the ileal digestibility of protein, increasing the amount of available N from the diet (Mc Alpine et al 2012, O'Shea et al 2014). Additional nutrients released could trigger a feedback mechanism to reduce feed intake because of a glucostatic and/or aminostatic response that could create nutrient imbalance in the gastrointestinal tract of pig (Nortey et al 2007).

\section{PLASMA UREA NITROGEN}

Decreasing dietary protein in pigs reduced the concentration of PUN (Qin et al 2015, Peng et al 2016). This lower PUN concentration is associated with a decrease in metabolic heat production associated with pig metabolism derived from lower amount of total nitrogen and hence, lower synthesis and excretion of urea originated by the AA excess when fed a standard CP diet, indicating better utilization of nitrogen by pigs fed LPD (Qin et al 2015). The increase in PUN concentration, which was observed in the present research when protease was added to the diet, may be due to greater digestibility of the CP diet and increased AA availability (Reyna et al 2006). The effect of the protease cannot be determined because reduction of protein never decreased growth performance of pigs. Thus, the extra amino acid available from the inclusion of the protease will be catabolized without further effect.

Based on the results of this study, we conclude that the reduction of the concentration of $\mathrm{AA}$ in the diet (low $\mathrm{CP}$ ) improves or maintains growth performance and reduce the plasma urea nitrogen in 25-100 kg hybrid barrows compared to standard CP diets, however, the lysine:AA ratio in the diet must be maintained. The addition of protected protease to pig diets does not show any effect on productive performance, because reduction of protein never decreased growth performance of pigs. 


\section{REFERENCES}

Adeola O, Cowieson AJ. 2011. Board-invited review: opportunities and challenges in using exogenous enzymes to improve non-ruminant animal production. J Anim Sci 89, 3189-3218.

AOAC, Association of Analytical Chemists. 2005. Official Methods of Analysis. $18^{\text {th }}$ ed. Association of Analytical Chemists, Washington DC, USA.

Burson D, Berg E. 2001. Procedures for estimating pork carcass composition. Pork quality facts. National Pork Producers Council, Des Moines IA, USA.

Chaney AL, Marbach EP. 1962. Modified reagents for determination of urea and ammonia. Clin Chem 8, 130-132.

Dubeau F, Julien PO, Pomar C. 2011. Formulating diets for growing pigs: economic and environmental considerations. Ann Oper Res 190, 239-269.

Figueroa JL, Estrada J, Zamora V, Cordero JL, Sánchez-Torres MT, et al. 2012. Digestible lysine levels in low-protein diets supplemented with synthetic amino acids for nursery, growing, and finishing barrows. Irish J Agric Food Res 51, 33-44.

Gallo L, Dalla Montà G, Carraro L, Cecchinato A, Carnier P, et al. 2014. Growth performance of heavy pigs fed restrictively diets with decreasing crude protein and indispensable amino acids content. Livest Sci 161, 130-138.

García E. 1988. Modificaciones al sistema de clasificación de Köppen (para adaptarlas a las condiciones de la República Mexicana). $4^{\text {ta }}$ ed. México DF.

Gloaguen M, Floc'h L, Corrent E, Primot Y, Van Milgen J. 2014. The use of free amino acids allows formulating very low crude protein diets for piglets. J Anim Sci 92, 637-644.

Gómez RS, Lewis AJ, Miller PS, Chen HY. 2002. Growth performance, diet apparent digestibility and plasma metabolite of barrows fed corn-soybean meal diets or low-protein, amino acid supplemented diets at different feeding levels. J Anim Sci 80, 644-653.

González M, Figueroa JL, Vaquera H, Sánchez-Torres MT, Ortega ME, et al. 2016. Metaanálisis del efecto de dietas bajas en proteína y adicionada con aminoácidos sintéticos para cerdos machos castrados en finalización. Arch Med Vet 48, 51-59.

Greiner L, Graham A, Knopf B, Ochoa L, Harrell RJ, et al. 2016. Effect of feeding protease at two soybean meal inclusion rates for nursery pigs. J Anim Sci 94, 117-117.

Guggenbuhl P, Waché Y, Wilson JW. 2012. Effects of dietary supplementation with a protease on the apparent ileal digestibility of the weaned piglet. J Anim Sci 90, 152-154.

He L, Wu L, Xu Z, Li T, Yao K, et al. 2016. Low-protein diets affect ileal amino acid digestibility and gene expression of digestive enzymes in growing and finishing pigs. Amino acids 48, 21-30.

Jiao X, Ma W, Chen Y, Li Z. 2016. Effects of amino acids supplementation in low crude protein diets on growth performance, carcass traits and serum parameters in finishing gilts. Anim Sci J 87, 1252-1257.

Karl RF, McDowell LR, Miles PH, Wilkinson NS, Funk JD, et al. 1979. Métodos de análisis de minerales para tejidos de plantas y animales. $2^{\text {da }}$ ed. Departamento de Ciencia Animal, Universidad de Florida, Gainesville, Florida, USA.

Mc Alpine PO, O'Shea CJ, Varley PF, O'Doherty JV. 2012. The effect of protease and xylanase enzymes on growth performance and nutrient digestibility in finisher pigs. J Anim Sci 90(Supplement 4), 375-377.

Morales MA, Cervantes M, Cuca M, Figueroa JL, Pro A, et al. 2002. Digestibilidad ileal de aminoácidos y comportamiento productivo de cerdos alimentados con dietas a base de trigo, adicionadas con una proteasa fungal. Agrociencia 36, 515-522.

Norma Oficial Mexicana, NOM-062-ZOO. 1999. Especificaciones técnicas para la producción, cuidado y uso de los animales de laboratorio. DOF-Segob, México.
Nortey TN, Patience JF, Simmins PH, Trottier NL, Zijlstra RT. 2007. Effects of individual or combined xylanase and phytase supplementation on energy, amino acid, and phosphorus digestibility and growth performance of grower pigs fed wheat-based diets containing wheat millrun. J Anim Sci 85, 1432-1443.

NRC, National Research Council. 2012. Nutrient requirements of swine. $11^{\text {th }}$ ed. National Academy Press. Washington DC, USA.

Orlando UAD, de Oliveira RFM, Donzele JL, Silva FCD, Generoso RAR, et al. 2007. Dietary crude protein levels and amino acid supplementation for gilts from 30 to $60 \mathrm{~kg}$ maintained in a high environmental temperature. Rev Bras Zootecn 36, 1573-1578.

Osada T, Takada R, Shinzato I. 2011. Potential reduction of greenhouse gas emission from swine manure by using a low-protein diet supplemented with synthetic amino acids. Anim Feed Sci Technol 166, 562-574.

O'Shea CJ, Mc Alpine PO, Solan P, Curran T, Varley PF, et al. 2014. The effect of protease and xylanase enzymes on growth performance, nutrient digestibility, and manure odour in grower-finisher pigs. Anim Feed Sci Tech 189, 88-97.

Pan L, Li P, Ma XK, Xu YT, Tian QY, et al. 2016. Tannin is a key factor in the determination and prediction of energy content in sorghum grains fed to growing pigs. J Anim Sci 29, 1761-1767.

Peng X, Hu L, Liu Y, Yan C, Fang ZF, et al. 2016. Effects of low-protein diets supplemented with indispensable amino acids on growth performance, intestinal morphology and immunological parameters in 13 to $35 \mathrm{~kg}$ pigs. Animal 10, 1812-1820.

Qin C, Huang P, Qiu K, Sun W, Xu L, et al. 2015. Influences of dietary protein sources and crude protein levels on intracellular free amino acid profile in the longissimus dorsi muscle of finishing gilts. J Anim Sci Biotechnol 6, 52.

Reyna L, Figueroa JL, Zamora V, Cordero JL, Sánchez-Torres MT, et al. 2006. Addition of protease to standard diet or low protein, amino acid-supplemented, sorghum-soybean meal diets for growing-finishing pigs. J Anim Vet Adv 12, 1202-1208.

Rostagno HS, Albino LFT, Hannas MI, Donzele JL, Sakomura NK, et al. 2017. Brazilian Tables for Poultry and Swine. Composition of feedstuffs and nutritional requirements. $4^{\text {th }}$ ed. Universidade Federal de Vicosa-Departamento de Zootecnia, Viçosa, Minas Gerais, Brasil.

SAS, Statistical Analysis System. 2010. SAS version 8.0. SAS Institute Inc., Cary, NC, USA.

Tous N, Lizardo R, Vilà B, Gispert M, Font-i-Furnols M, et al. 2014. Effect of reducing dietary protein and lysine on growth performance, carcass characteristics, intramuscular fat, and fatty acid profile of finishing barrows. J Anim Sci 92, 129-140.

Wang D, Piao XS, Zeng ZK, Lu T, Zhang Q, et al. 2011. Effects of keratinase on performance, nutrient utilization, intestinal morphology, intestinal ecology and inflammatory response of weaned piglets fed diets with different levels of crude protein. Asian Australas J Anim Sci 24, 1718-1728.

Xu X, Wang H, Pan L, Ma XK, Tian QY, et al. 2017. Effects of coated proteases on the performance, nutrient retention, gut morphology and carcass traits of broilers fed corn or sorghum based diets supplemented with soybean meal. Anim Feed Sci Technol 223, 119-127.

Yu GX, Chen DW, Yu B, He J, Zheng P, et al. 2016. Coated protease increases ileal digestibility of protein and amino acids in weaned piglets. Anim Feed Sci Technol 214, 142-147.

Zamora V, Figueroa JL, Reyna L, Cordero JL, Sánchez-Torres MT, et al. 2011. Growth performance, carcass characteristics and plasma urea nitrogen concentration of nursery pigs fed low-protein diets supplemented with glucomannans or protease. J ApplAnim Res 39, 53-56.

Zuo J, Ling B, Long L, Li T, Lahaye L, et al. 2015. Effect of dietary supplementation with protease on growth performance, nutrient digestibility, intestinal morphology, digestive enzymes and gene expression of weaned piglets. Anim Nutr 1, 276-282. 